\title{
The effect of the vessel wall motion on color flow mapping images
}

\author{
Qinghui Zhang ${ }^{1,2, a}$, Yufeng Zhang ${ }^{1, b^{*}}$, Yi Zhou ${ }^{1, c}$, Lian Gao ${ }^{1, d}$ \\ ${ }^{1}$ Department of Electronic Engineering, Information School, Yunnan University, Kunming, Yunnan \\ 650091, China \\ ${ }^{2}$ School of Computer and Information, Southwest Forestry University, Kunming, Yunnan 650224, \\ China \\ ahuizq@163.com, byfengzhang@yahoo.com, czhouyikm@gmail.com, d962245641@qq.com
}

Keywords: Vessel wall motion; Pulsatile flow; Stenosis; Ultrasound; CFM

Abstract. This study focus on the effects of motions of vessel wall on the color flow mapping (CFM) images and whether the CFM images can truthfully reflect the behaviors of the vessel wall and blood flow. By coupling numerical Runge-Kutta and ultrasound simulation method, a model is proposed to generate CFM images, considering wall vibration, vessel stenosis and blood pulsatility. The cases with six different wall oscillation frequencies and four different stenosis degrees are investigated. The results show that the motion of vessel wall has effect on CFM images, and the generated CFM images will reflect the motion of vessel wall. Thus, this simulation model can be used as a tool to assess the interaction between the vessel wall and blood flow motion, and to evaluate ultrasound signal processing and visualization techniques.

\section{Introduction}

Cardiovascular disease, which is related to vessel stenosis, is one of the most common causes of death in the world. Therefore, an early diagnosis and treatment is critical. Thus, many researchers created simulation models to imitate the behaviors of the vessel wall and pulsatile blood flow [1-3]. The effects of mechanical parameters of the elastic vessel such as stenosis severity, stenosis geometry, and length on the flow field are studied [4-6]. However, the effect of the vessel wall on blood flow is not addressed in these researches. CFM technology has been used as one of the most important non-invasive diagnostic methods for detection and monitoring of cardiovascular disease. However, the ultrasound images cannot always accurately demonstrate the behaviors of vessel wall and blood flow. Thus, the main aim of this work focus on the effect of vessel wall motion on the CFM images and whether the CFM images can truthfully reflect the behaviors of the vessel wall, and blood flow.

\section{Method}

We firstly set up the geometry model of a stenotic vessel, and define the physiological and geometrical parameters, such as stenosis width, radius of artery, fundamental frequency of blood flow based on clinical data. Secondly, we get the numerical solutions of Navier-Stokes and vessel wall equations which describe the behaviors of the blood flow through the stenotic vessel. And then, these calculated numerical values are used as input of the Field II (an ultrasound field simulation software) [7]. Finally, the CFM images are generated and compared with numerical results.

$$
R(x)=\left\{\begin{array}{lr}
R_{0}\left[1-\frac{\delta}{2 R_{0}}(1+\sin \omega t)\left(1+\cos \frac{\pi x}{x_{0}}\right)\right] & x \in\left[-x_{0}, x_{0}\right] \\
R_{0} & \text { otherwise }
\end{array}\right.
$$

The geometry of the stenotic vessel is with an axisymmetric cosine shaped plaque. The mathematical description of the vessel radius is denoted as Eq. 1 with the cylindrical polar coordinate system. Where $R(x)$ and $R_{0}$ are the radius of the narrowed and normal vessel respectively, $\delta$ is stenosis severity, $x_{0}$ is the half length of the stenosis. In this study, six different wall vibration frequencies 
$\left(0 * \omega_{0}, 1 * \omega_{0}, 1.25 * \omega_{0}, 1.5 \omega_{0}, 1.75 \omega_{0}, 2 * \omega_{0}\right)$ and four different stenosis degrees $(10 \%, 15 \%, 20 \%$, $25 \%$ ) have been studied, where $\omega_{0}$ is fundamental frequency of blood. Because the blood is assumed as Newtonian, laminar, fully developed, viscous and incompressible periodically pulsatile flow, the linearized Navier-Stokes and wall motion can be expressed by a second-order differential equation:

$$
\frac{1}{n^{2} \omega_{0}^{2} \rho}\left(\left(\frac{R}{2}-\frac{1}{\beta_{n} j^{\frac{3}{2}}} \frac{J_{1}\left(\beta_{n} j^{\frac{3}{2}} R\right)}{J_{0}\left(\beta_{n} j^{\frac{3}{2}} R\right)}\right) \frac{d^{2} p_{n}}{d x^{2}}+\frac{d R}{d x} \frac{J_{1}^{2}\left(\beta_{n} j^{\frac{3}{2}} R\right)}{J^{2}{ }_{0}\left(\beta_{n} j^{\frac{3}{2}} R\right)} \frac{d p_{n}}{d x}\right)+\frac{R^{2}}{B h} p_{n}=0
$$

Where $p_{n}$ is $n$-th harmonic item of blood pressure; $\beta n=\sqrt{\rho \omega_{0} n / \eta}, n=1,2,3, L ; J_{0}$ and $J_{1}$ are first kind Bessel functions with zero-order and first-order, respectively; $B=E /\left(1-\sigma^{2}\right), E$ is Young's modulus, $\sigma$ is passion ratio. The Runge-Kutta method is used to solve this equation. Then the axial velocity profiles of blood flow in the narrowed vessel with different oscillation frequencies and stenosis degrees are obtained. By treating tissues as a collection of randomly distributed point scatterers moving with velocities, we can update the positions of the scatterers at any time with the calculated velocities. Then, with help of Field II software, we obtain the CFM images.

\section{Results and Discussion}


Stenosis degree $=10 \%$
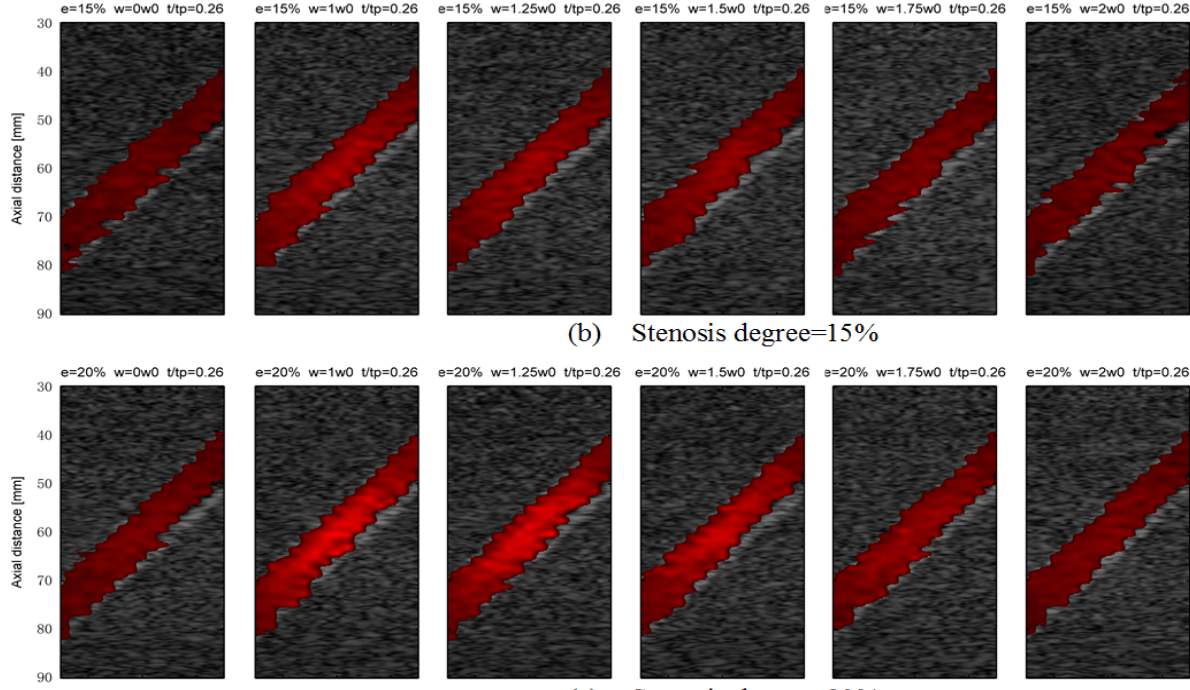

(b) Stenosis degree $=15 \%$
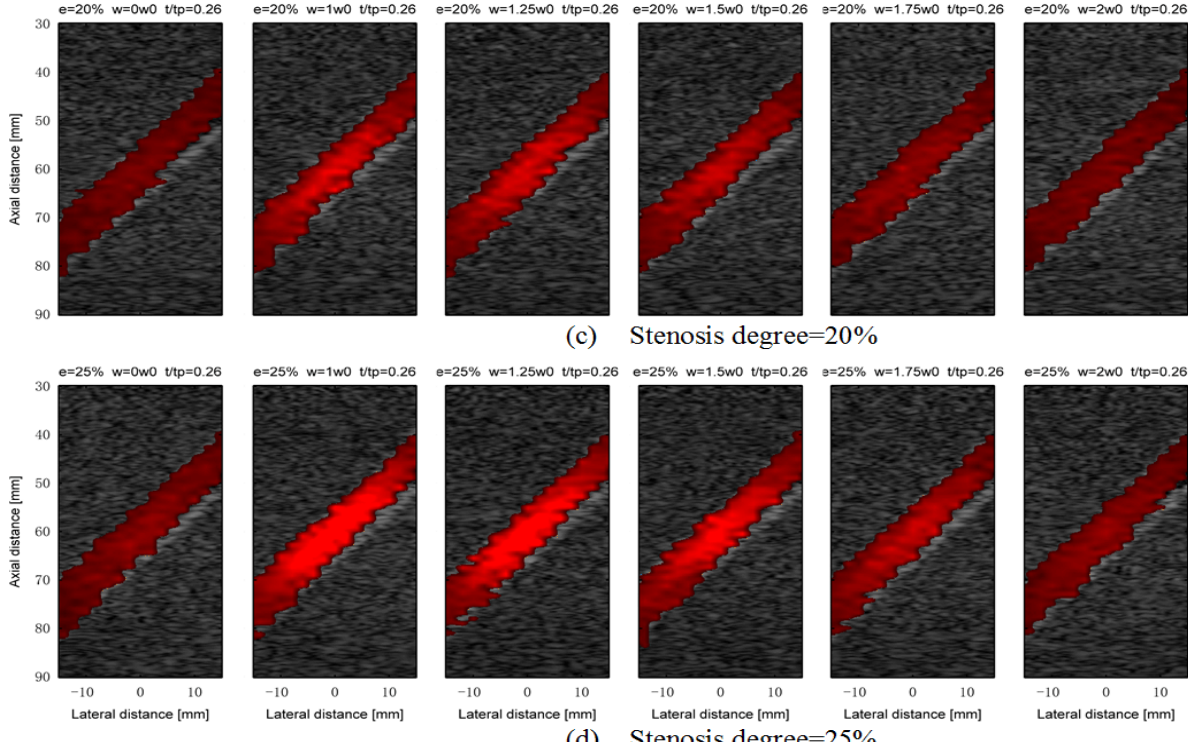

(c) Stenosis degree $=20 \%$

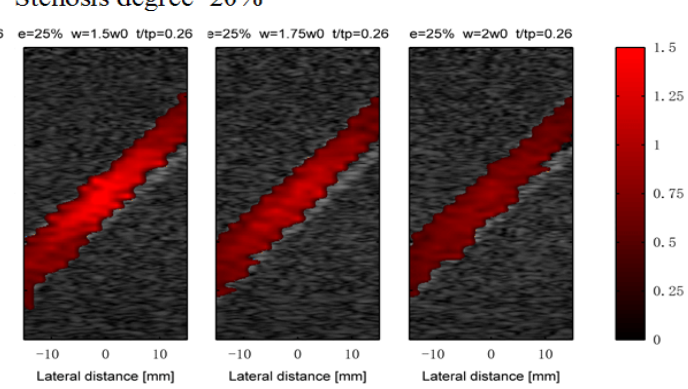

(d) Stenosis degree $=25 \%$

Fig. 1. Simulated CFM images with stenosis degrees of (a) $10 \%$, (b) $15 \%$, (c) $20 \%$, (d) $25 \%$ and six different wall oscillating frequencies $\left(0 * \omega_{0}, 1 * \omega_{0}, 1.25 * \omega_{0}, 1.5 \omega_{0}, 1.75 \omega_{0}, 2 * \omega_{0}\right)$ 
Spatial analysis with CFM simulation. Undoubtedly, the motion of vessel wall motion will have an impact on blood flow. As shown in Fig. 1, CFM images of the systolic acceleration phase are generated to help investigate the modulation effects of the vessel wall with stenosis on the flow field. By comparing, we learn that the blood in subfigures with wall vibration is redder than that without wall vibration (the first column, $\omega=0 * \omega_{0}$ ). This means that the highest value of the blood velocity in vessel with wall vibration is higher than that without wall vibration. The reason is that the oscillating motion will increase the degree of stenosis, which leads to the increase of blood pressure and result in increase of blood velocity. Furthermore, the highest estimated velocity of blood decrease while the frequency of wall vibration increase. Moreover, by comparing between the rows, the increased value of estimated blood velocity with higher stenosis degree is much striking than that with lower degrees.

Temporal analysis. For quantitative evaluating the effect of wall vibrating frequency and stenosis degrees on blood flow, comparisons among six different frequencies and four stenosis degrees are illustrated in Fig. 2 and Fig. 3. The radial and axial central point in vessel are chosen to demonstrate the comparison. Overall, the difference of the vessel wall vibration frequency and stenosis degree will change the velocity of blood flow, and the blood behaviors modulated by wall motion show good agreement with CFM results shown in Fig. 1. Fig. 2 illustrate the effect of wall vibrating frequency on blood velocity. It can be obtained that the closer to blood fundamental frequency the wall vibrating frequency is, the higher peak velocity becomes. In addition, the velocity change caused by wall vibration is unobvious when the stenosis is small. However, the change becomes very significant when the stenosis degree is high. For example, as Fig. 2(a) shown, the peak velocity of blood in vessel with $l^{*} \omega_{0}$ wall vibrating frequency is just about 1.27 times of that without vibration, while, as Fig. 2(d) shown (25\% stenosis degree), that of blood with $1 * \omega_{0}$ wall vibrating frequency almost triples. Fig. 3 focus on the influence on blood velocity caused by stenosis. By comparing, we learn that the higher stenosis degree, the higher velocity of blood. Similarly, the velocity change caused by stenosis is unobvious when the difference between wall vibration frequency and blood fundamental frequency is big. However, the changes become significant when the difference is small. These results coincide with that from Fig. 2. The peak velocities under different situation are summarized in Table 1.

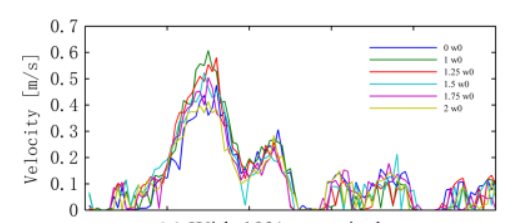

(a) With $10 \%$ stenosis degree

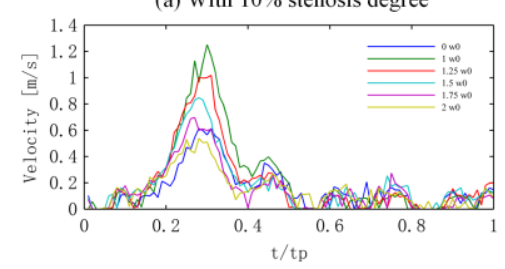

(c) With $20 \%$ stenosis degree

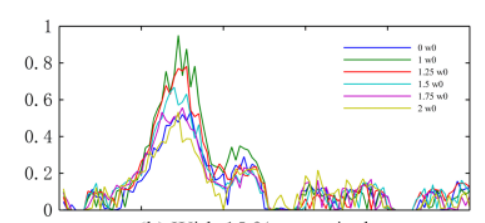

(b) With $15 \%$ stenosis degree

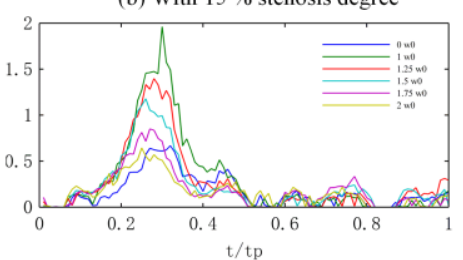

(d) With $25 \%$ stenosis degree

Fig. 2. Comparison of velocities with six different wall vibrating frequencies and same stenosis degree

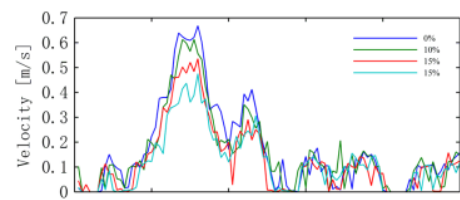

(a) $\omega=0 * \omega_{0}$

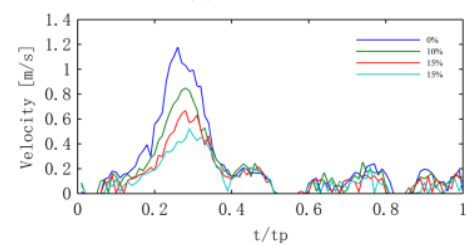

(d) $\omega=1.5 * \omega_{0}$

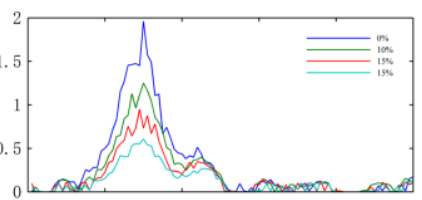

(b) $\omega=1 * \omega_{0}$

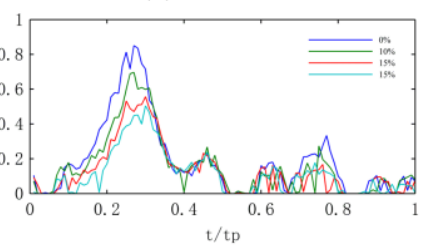

(e) $\omega=1.75 * \omega_{0}$

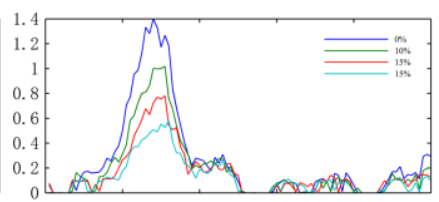

(c) $\omega=1.25 * \omega_{0}$

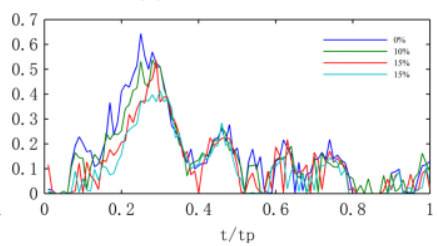

(f) $\omega=2 * \omega_{0}$

Fig. 3. Comparison of velocities with four different stenosis degrees and same wall vibrating frequency 
Table 1: Peak velocity with six wall vibrating frequencies and four stenosis degree

\begin{tabular}{cccccccc}
\hline & \multicolumn{6}{c}{ Wall vibrating frequency $(\omega)$} \\
Peak velocity $(\mathrm{m} / \mathrm{s})$ & $0 * \omega$ & $1 *^{*} \omega$ & $1.25 * \omega$ & $1.5 * \omega$ & $1.75 * \omega$ & $2 * \omega$ \\
& $10 \%$ & 0.48 & 0.61 & 0 & 0 & 0 & 0 \\
\hline \multirow{5}{*}{ Stenosis degree } & $15 \%$ & 0.54 & 0.95 & 0.78 & 0.52 & 0.50 & 0.41 \\
& $20 \%$ & 0.61 & 1.25 & 1.02 & 0.85 & 0.56 & 0.53 \\
& $25 \%$ & 0.67 & 1.96 & 1.40 & 1.18 & 0.85 & 0.64 \\
\hline
\end{tabular}

In order to examine the accuracy of estimated velocity generated by ultrasound method, the comparison to velocity generated by Runge-Kutta method is demonstrated in Fig. 4. As shown, there is small difference between the two type curves. Further observation shows the difference of higher velocity is seemly bigger than lower one. For quantitative analysis, the residual sum of squares of estimated velocity with different wall vibrating frequencies and stenosis degrees are calculated and listed in Table 2. The maximal residual sum of squares reach $1.12 \%$ when $\omega$ is $0^{*} \omega_{0}$ and stenosis degree is $25 \%$.
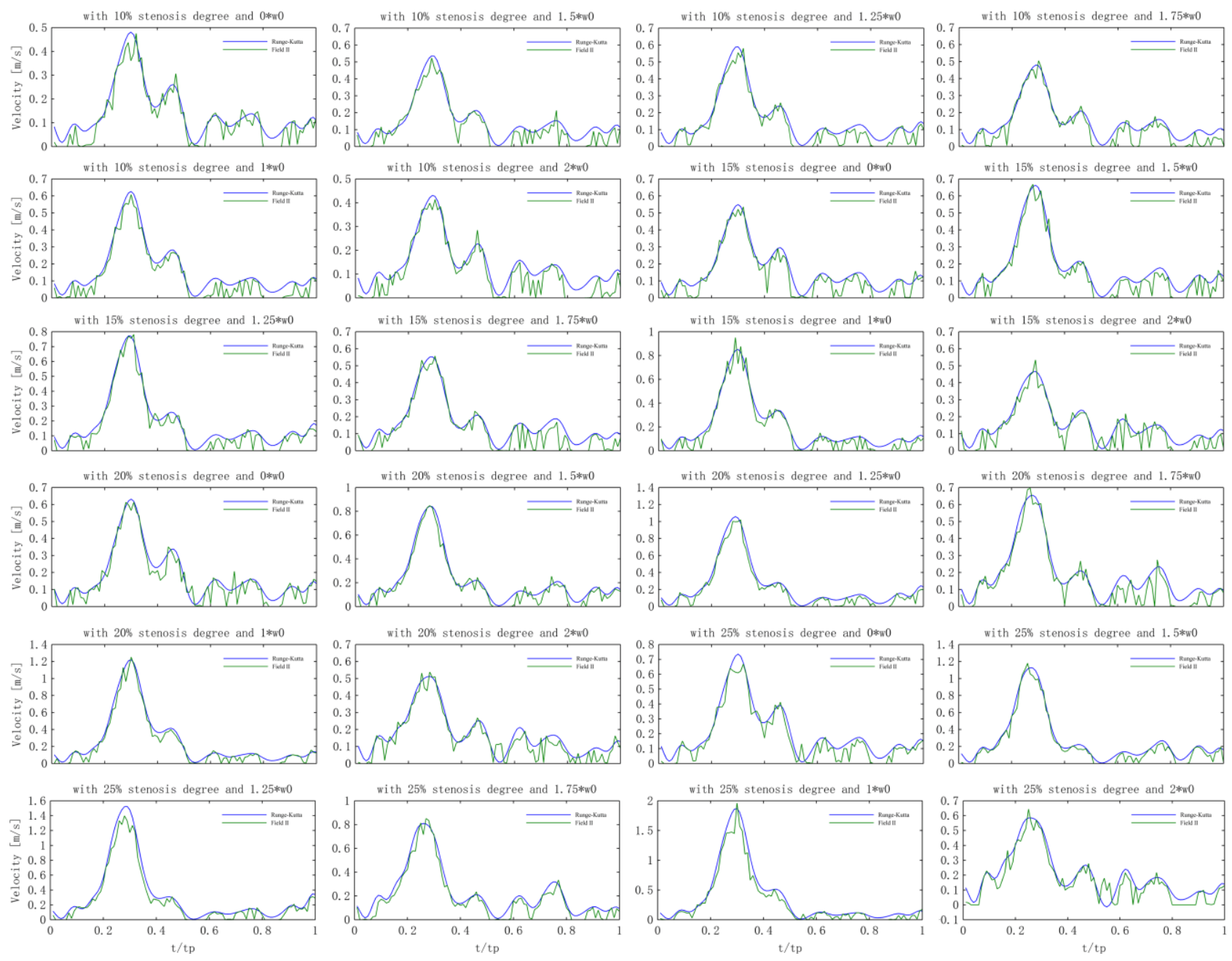

Fig. 4. Comparison between velocities generated by Runge-Kutta and ultrasound method

\section{Conclusion}

This study focus on the effects of motions of vessel wall on the CFM images and whether the CFM images can truthfully reflect the behaviors of the vessel wall and blood flow. A simulation model is proposed to generate images, considering wall vibration, vessel stenosis and blood pulsatility. The 
cases with six different wall oscillation frequencies and four different stenosis degrees are investigated. By comparing, it can be concluded from the results that the motion of vessel wall has significantly effect on these CFM images, and the generated CFM images will reflect the motion of vessel wall. Thus, this type of simulation model can be used as an effective tool to assess the interaction between the vessel wall and blood flow motion, and to further evaluate ultrasound signal processing and visualization techniques.

Table 2: Residual sum of squares of estimated velocity

\begin{tabular}{cccccccc}
\hline \hline & & \multicolumn{7}{c}{ Wall vibrating frequency $(\omega)$} \\
Residual sum of squares & $0 * \omega_{0}$ & $1 * \omega_{0}$ & $1.25 * \omega$ & $1.5 * \omega$ & $1.75 * \omega$ & $2 * \omega_{0}$ \\
& & & 0 & 0 & 0 & \\
\hline \multirow{3}{*}{ Stenosis degree } & $10 \%$ & $0.18 \%$ & $0.24 \%$ & $0.19 \%$ & $0.24 \%$ & $0.22 \%$ & $0.22 \%$ \\
& $20 \%$ & $0.24 \%$ & $0.28 \%$ & $0.20 \%$ & $0.23 \%$ & $0.27 \%$ & $0.28 \%$ \\
& $25 \%$ & $0.30 \%$ & $1.12 \%$ & $0.67 \%$ & $0.31 \%$ & $0.30 \%$ & $0.35 \%$ \\
\hline
\end{tabular}

\section{References}

[1] C. Cheng, D. Tempel, R. van Haperen, A. van der Baan, F. Grosveld, M. J. Daemen, et al., "Atherosclerotic lesion size and vulnerability are determined by patterns of fluid shear stress," Circulation, vol. 113, pp. 2744-2753, 2006.

[2] Y. Fukumoto, T. Hiro, T. Fujii, G. Hashimoto, T. Fujimura, J. Yamada, et al., "Localized elevation of shear stress is related to coronary plaque rupture: a 3-dimensional intravascular ultrasound study with in-vivo color mapping of shear stress distribution," Journal of the American College of Cardiology, vol. 51, pp. 645-650, 2008.

[3] G. Zendehbudi and M. Moayeri, "Comparison of physiological and simple pulsatile flows through stenosed arteries," Journal of Biomechanics, vol. 32, pp. 959-965, 1999.

[4] B. Buriev, T. Kim, and T. Seo, "Fluid-structure interactions of physiological flow in stenosed artery," Korea-Australia Rheology Journal, vol. 21, pp. 39-46, 2009.

[5] T. Belzacq, S. Avril, E. Leriche, and A. Delache, "A numerical parametric study of the mechanical action of pulsatile blood flow onto axisymmetric stenosed arteries," Med Eng Phys, vol. 34, pp. 1483-95, Dec 2012.

[6] A. Swillens, P. Segers, and L. Lovstakken, "Two-dimensional flow imaging in the carotid bifurcation using a combined speckle tracking and phase-shift estimator: a study based on ultrasound simulations and in vivo analysis," Ultrasound Med Biol, vol. 36, pp. 1722-35, Oct 2010.

[7] J. A. Jensen, "Field: A program for simulating ultrasound systems," in 10TH NORDICBALTIC CONFERENCE ON BIOMEDICAL IMAGING, VOL. 4, SUPPLEMENT 1, PART 1: 351--353, 1996. 